\title{
Carra Câncer de tireoide no Brasil: o que dizem e o que não dizem os Registros Hospitalares de Câncer
}

doi: 10.1590/S1679-49742021000300025

\section{Thyroid cancer in Brazil: what the Hospital Cancer Records say and what they don't say \\ Cáncer de tiroides en Brasil: lo que dicen y lo que no dicen los registros hospitalarios de cáncer}

\author{
Eliane de Freitas Drumond ${ }^{1}$ - (1) orcid.org/0000-0003-2159-4250 \\ Maria Cristina Ferreira Drummond ${ }^{2}$ - (1) orcid.org/0000-0003-1801-9319 \\ 'Instituto Mário Penna, Núcleo de Ensino e Pesquisa, Belo Horizonte, MG, Brasil \\ ${ }^{2}$ Instituto Mário Penna, Ambulatório de Endocrinologia e Metabologia Oncológica, Belo Horizonte, MG, Brasil
}

Prezados Editores,

Os resultados do artigo de Borges et al., ${ }^{1}$ intitulado 'Câncer de tireoide no Brasil: estudo descritivo dos casos informados pelos registros hospitalares de câncer, 2000-2016', publicado no fascículo 4 do volume 29 da RESS, mostram as potencialidades dos registros hospitalares de câncer (RHC) no delineamento de perfis de diagnóstico e tratamento do paciente com neoplasia de tireoide no Brasil; e também deixam transparecer as fragilidades desse sistema, implantado na rede de atenção ao paciente oncológico habilitada pelo Sistema Único de Saúde (SUS).

Em consonância com a literatura nacional e internacional, ${ }^{2,3,4} 0$ referido estudo evidenciou aumento nos casos de câncer de tireoide no país, ainda que 3.482 casos $(6,2 \%)$ tenham sido excluídos dos 56.394 potencialmente elegíveis por ausência da informação sobre tipo histológico. 0s baixos percentuais de incompletude $(<5 \%)$ das variáveis 'sexo', 'idade', 'diagnóstico e tratamento anteriores' e 'Unidade da Federação' de residência e de tratamento permitiram observar: (i) a maior razão de sexos; (ii) a distribuição etária e 0 tipo de tumor ( 45 a 55, 50 e 60 anos, para carcinoma diferenciado, medular e anaplásico, respectivamente); e (iii) a migração dos indivíduos em busca de atendimento como causa do atraso de seu tratamento.

No estudo, a elevada incompletude do estadiamento do tumor foi reduzida a 39,6\%, utilizando-se a correlação entre o estadiamento e a TNM (em inglês, Classification of Malignant Tumors, ou Classificação de Tumores Malignos). 0 estadiamento do tumor é, reconhecidamente, a variável definidora do tratamento mais adequado e do prognóstico; entretanto, o método para redução utilizado no trabalho não é descrito com suficiente clareza, de maneira a permitir sua replicação.

Destaca-se, outrossim, que não há descrição, nos métodos do estudo, de como foi obtida a informação do tratamento de radioiodoterapia, uma vez que essa

Endereço para correspondência:

Eliane de Freitas Drumond - Rua Dona Cecília, n 500, Apt. 1403, Serra, Belo Horizonte, Minas Gerais, Brasil. CEP: 30220-070

E-mail: elianedrumond1@gmail.com 
opção terapêutica não está disponível no RHC. Apenas a radioterapia é contemplada. Ao se considerarem as duas modalidades equivalentes, há o risco de interpretações inadequadas, por se tratar de terapias diversas, tanto no que se refere à indicação quanto às finalidades terapêuticas, ambas dependentes de variáveis e especificidades que diferem nos três grupos de tumores analisados.

Os autores do manuscrito ressaltam a necessidade do preenchimento adequado dos prontuários assistenciais, fontes de dados para planejamento, controle e avaliação da rede de atenção ao paciente oncológico, a que acrescentaríamos o fato de algumas das limitações observadas decorrerem de problemas nos RHCs. Poder-se-ia apontar, por exemplo, a necessidade de revisão da Ficha de Registro de Tumor, especialmente no que se refere a modalidades terapêuticas - no caso, das neoplasias de tireoide - e atualizações da classificação TNM, à medida de sua publicação.

\section{Referências}

1. Borges AKM, Ferreira JD, Koifman S, Koifman RJ. Câncer de tireoide no Brasil: estudo descritivo dos casos informados pelos registros hospitalares de câncer, 2000-2016. Epidemiol Serv Saúde. 2020;29(4):e2019503. https://doi. org/10.5123/s1679-49742020000400012.

2. Haugen BR, Alexander EK, Bible KC. 2015 American thyroid association management guidelines for adult patients with thyroid nodules and differentiated thyroid cancer: the american thyroid association guidelines task force on thyroid nodules and differentiated thyroid cancer. Thyroid. 2016;26(1):1133. doi: http://doi.org/10.1089/thy.2015.0020.
3. Filetti S, Durante C, Hartl D, Leboulleux S, Locati LD, Newbold K, et al. Thyroid cancer: ESMO Clinical Practice Guidelines for diagnosis, treatment and follow-up. Ann Oncol. 2019;30(12):1856-83. doi: http://doi.org/10.1093/annonc/mdz400.

4. Pacini F, Basolo F, Bellantone R, Boni G, Cannizzaro $\mathrm{M}$, De Palma $\mathrm{C}$, et al. Italian consensus on diagnosis and treatment of differentiated thyroid cancer: joint statements of six Italian societies. J Endocrinol Invest. 2018;41(7):849-76. doi: http://doi.org/10.1007/s40618-018-0884-2. 